\title{
Suppression of Electromagnetic Interference using Screening and Shielding Techniques within Switching Cells
}

\author{
Zhe Zhang \\ Power electronics, Machines and control group, electrical \\ engineering, University of Nottingham, UK, NG72RD \\ Zhe.zhang@nottingham.ac.uk
}

\author{
Mark Johnson \\ Power electronics, Machines and control group, electrical \\ engineering, University of Nottingham, UK, NG72RD \\ Mark.johnson@nottingham.ac.uk
}

\begin{abstract}
- in this paper we introduce the use of combination of screening and shielding to suppress electromagnetic interference (EMI) generated by a switching cell. We investigate the screening of common mode (CM) currents and the shielding of magnetic fields generated by a model switching cell using a combination of simulation tools and measurements. Simulation results align with measurements very well up to $100 \mathrm{MHz}$, confirming that a relatively simple model can be used to investigate different arrangements of screen layer. We demonstrate that electromagnetically coupled currents produced by rapidly changing voltages and currents in the main switching circuit can be circulated back to the local DC-link or absorbed rather than being coupled to the external environment.
\end{abstract}

Keywords-Wide Band Gap device; EMI; screening; shielding

\section{INTRODUCTION}

Wide Band Gap (WBG) devices and power modules are enablers to meet new requirements for high power density and low power loss. In recent research, WBG devices fabricated from Silicon Carbide ( $\mathrm{SiC})$ and Gallium Nitride $(\mathrm{GaN})$ have realized high switching speeds enabling high efficiency, high frequency switching. However, high $\mathrm{dv} / \mathrm{dt}$ at the output terminals of inverters causes massive Differential Mode (DM) noise to circulate between the inverter and load, leading to motor winding stresses due to capacitively-coupled currents and voltage reflections. At the same time, rapidly changing voltages can cause massive Common Mode (CM) noise to circulate through the ground path back to the power module. This CM noise may introduce power losses and system malfunctions, such as deterioration of the motor winding insulation and high ground leakage currents from the motor windings. In addition, the power converter together with its associated connections to the power source and load can become a strong source of radiated EMI, which can then couple with nearby electrical systems.

Electromagnetic interference arises from the rapid voltage and current transitions occurring during operation of the switching cell. A simplified model of the sources of EMI, the propagation path and a typical measurement arrangement for conducted EMI is showed in Figure.1. $V n$ is a noise source representing the switching node of the converter, $\mathrm{Cng}$ is the parasitic capacitance between the switching node and ground.
The circulation paths for differential-mode and common-mode EMI are shown respectively by the green and magenta lines. So far, many researchers have focused on how to model the noise source [1] and how to design better CM and DM filters [2] [3] outside the switching cell to suppress EMI noise by introducing high impedance filter configurations to block EMI noise and using low impedance filter configuration to shunt noise back to the source. Other researchers have tackled EMI problems inside power modules by reducing either parasitic inductance [4] [5], due to interconnections, or parasitic capacitance to "ground" through heatsink [6].

In contrast to the conventional approach of tackling EMI, we propose an alternative approach, utilizing a combination of screening and shielding to solve EMI problems within the switching cell. The schematic of Figure. 2 illustrates the function of the screen in suppression of the common mode current that would otherwise circulate in the ground path. The screen is electrically connected to the switching cell DC-link decoupling capacitance, providing a path for local recirculation of common mode currents which are coupled electromagnetically into screen. Differential mode noise can be suppressed by incorporating a low-pass filter, which may also be integrated within the switching cell. In this case, the screen can provide a convenient means for returning the high-frequency noise to the source. The screen will also attenuate radiated electromagnetic fields, as long as it is significantly thicker than the electromagnetic skin-depth at the converter switching frequency. In this paper we discuss the modeling and behavior of shielding and screening layers that are applicable to switching cells.

\section{THEORY}

\section{A. CM current screening}

Figure. 3 shows an approximate, small-signal, ac-equivalent circuit for the switching cell, screen and ground return path. $V n$ is the switching noise source, $V s$ is the screen voltage and $V g$ is the ground voltage. The voltage definitions for the screen and ground are not intended to correspond to any particular geometrical arrangement and are included solely to aid the analysis. Voltages and impedances for this arrangement will in general be distributed with variations expected across the bus, 
screen and ground planes. Cns represents the capacitance from noise source to screen, $C s g$ represents the capacitance from screen to ground and $\mathrm{Cng}$ represents the capacitance from noise source to ground. $Z s(Y s)$ is the screen return path impedance (admittance), $Z g(Y g)$ is the ground return path impedance (admittance).

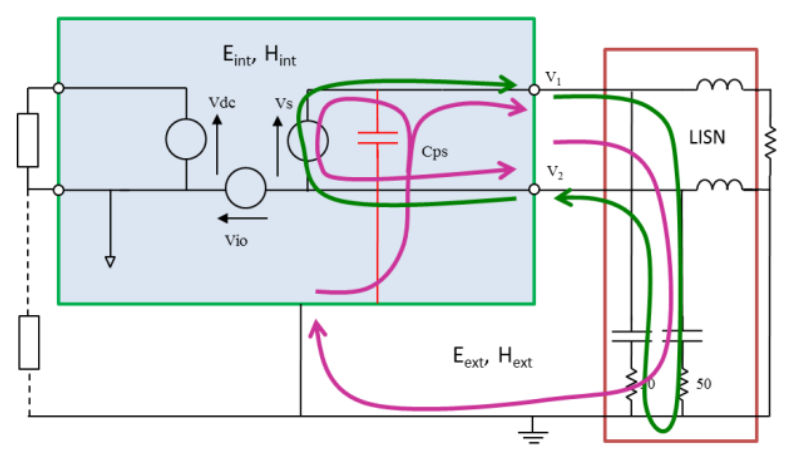

Fig.1: simplified equivalent circuit EMI model for a switching cell.

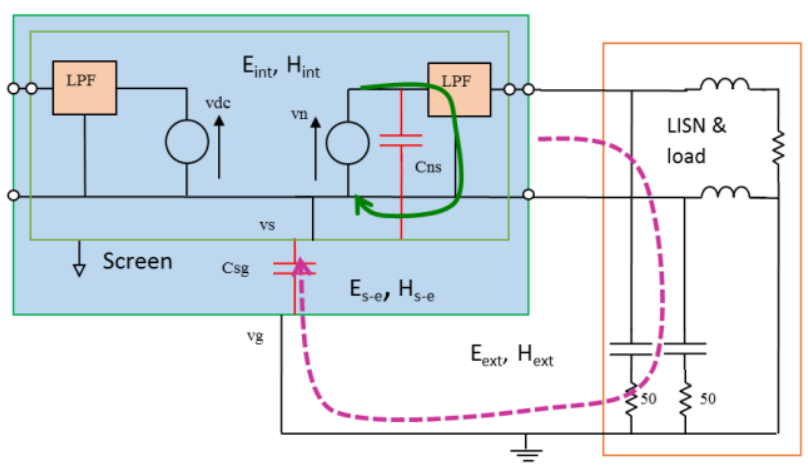

Figure 2. Simplified equivalent circuit of integrated switching cell with EMI suppression features.

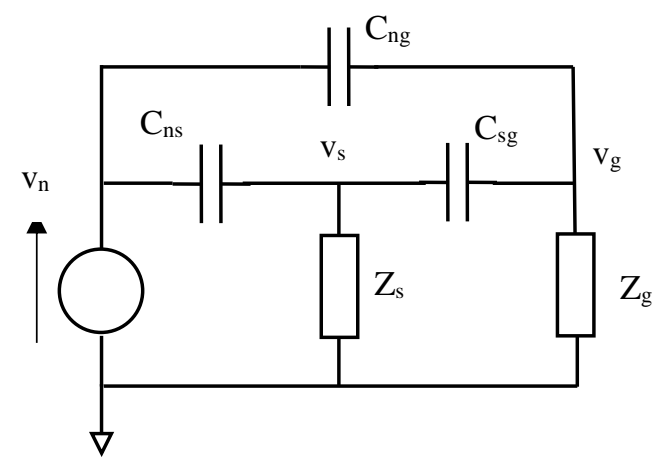

Figure.3 Approximate equivalent circuit for common-mode analysis.

Following equations obtained from Nodal analysis:

$v_{g}\left(Y_{n g}+Y_{s g}+Y_{g}\right)=v_{n} Y_{n g}+v_{s} Y_{s g}$

$v_{s}\left(Y_{n s}+Y_{s g}+Y_{s}\right)=v_{n} Y_{n s}+v_{g} Y_{s g}$
Eliminating $\mathrm{v}_{\mathrm{s}}$ :

$$
\begin{aligned}
& v_{g}\left(\left(Y_{n g}+Y_{g}\right)\left(Y_{n s}+Y_{s g}+Y_{s}\right)+Y_{s g}\left(Y_{n s}+Y_{s}\right)\right)= \\
& v_{n}\left(Y_{n g}\left(Y_{n s}+Y_{s g}+Y_{s}\right)+Y_{n s} Y_{s g}\right)
\end{aligned}
$$

From which we can obtain the common-mode current:

$$
i_{g}=v_{n} Y_{g} \frac{Y_{n g}\left(Y_{n s}+Y_{s g}+Y_{s}\right)+Y_{n s} Y_{s g}}{\left(Y_{n g}+Y_{g}\right)\left(Y_{n s}+Y_{s g}+Y_{s}\right)+Y_{s g}\left(Y_{n s}+Y_{s}\right)}
$$

Some specific cases:

1) No screen: $Y_{n s}=Y_{s g}=0$

$$
i_{g}=v_{n} Y_{g} \frac{Y_{n g}}{\left(Y_{n g}+Y_{g}\right)}
$$

At high frequencies $Y_{n g}$ will dominate and the current will be limited by $\mathrm{Y}_{\mathrm{g}}$ for example the high impedance presented by a $\mathrm{CM}$ filter, which is a common solution to attenuate noise current coupled from output to ground.

2) Screen and no direct coupling from source to ground:

$$
\begin{aligned}
& Y_{n g}=0 \\
& i_{g}=v_{n} Y_{g} \frac{Y_{n s} Y_{s g}}{Y_{g}\left(Y_{n s}+Y_{s g}+Y_{s}\right)+Y_{s g}\left(Y_{n s}+Y_{s}\right)}
\end{aligned}
$$

a) $Y_{s} \gg Y_{n s}, Y_{s g}$

$$
i_{g}=v_{n} Y_{g} \frac{Y_{n s}}{Y_{s}\left(\frac{Y_{g}}{Y_{s g}}+1\right)}
$$

At high frequencies the CM current is determined by the ratio $Y_{n s} /_{Y_{s}}$ (assuming $Y_{g}$ is small).

b) $Y_{s} \ll Y_{n s}, Y_{s g}$

$$
i_{g}=v_{n} Y_{g} \frac{Y_{n s} Y_{s g}}{Y_{g}\left(Y_{n s}+Y_{s g}\right)+Y_{n s} Y_{s g}}
$$

This case is the same as the unscreened case but with the series combination of capacitors $Y_{n s}, Y_{s g}$. From case 2), we can understand that in the case when the screen can completely decouple noise source from ground, CM current is greatly determined by the impedance of return path. As illustrated by equation. (7), with careful design of return path and screen layer, the CM current can be significantly attenuated. However, if at high frequency the return path becomes high impedance, the CM current couples to ground through the screen layer as described in equation (8).

3) Screen and direct coupling from source to ground, $Y_{s} \gg Y_{n s}, Y_{s g}$ : 


$$
i_{g}=v_{n} Y_{g} \frac{Y_{n g}}{\left(Y_{n g}+Y_{s g}+Y_{g}\right)}
$$

The "leakage" of electric flux around the shield limits its performance: $Y_{n g}$ should be kept to a minimum. In all cases $Z s$ is a critical element impacting screening effectiveness. A small $Z s$ ensures the electromagnetically coupled current can be recirculated back to the noise source, and minimizes the component of current flowing in the ground return path.

\section{B. Magnetic field shielding}

Due to very fast changing currents circulating inside the switching cell, fast changing electromagnetic fields will be generated outside the cell, leading to unintentional electromagnetic interference (EMI) problem. Besides reducing the circulating current loop which can relatively reduce radiative EMI noise, metal shield layers are a good option to effectively stop EMI wave from propagating outside power converter by generating eddy currents on the surface of metal shield layer. Incident magnetic fields induce current in a shorted conductive loop and the current in turn generates counter fields that effectively cancel the incident fields. The magnetic flux produced by the eddy currents has components 90 degree out of phase with the incident flux. This in turn will cause an induced electromagnetic force (EMF) opposing the original current excitation causing an increase of resistance and decrease of selfinductance in the originating circuit.

The shield thickness required in order to generate ample amount of eddy currents for proper field cancellation is directly proportional to the skin depth, $\delta$, which decreases with increasing frequency. When a magnetic field generated by a current-carrying conductor comes in contact with a material of near-infinite conductivity, then skin depth will become vanishingly small, and we can consider it approaches $\delta \approx 0$. In this case eddy currents only flow at the surface of shield layer. Magnetic field strength inside shield layer is infinitely small, no magnetic field can penetrate through the metal shield layer and all magnetic fields near shield layer are repelled (or diverted) away from shield layer, tangentially along the surface of shield layer.

\section{SIMULATIONS AND MEASUREMENTS}

\section{A. CM screening}

In order to relate this concept to a practical case and to illustrate the behavior of the screen layer, we use a simple model within ANSYS MAXWELL, shown in Fig.5, which includes the major noise source, shield layer, ground and return point. This simple model can well represent most cases of switching cell such as a half bridge. A copper sheet of size $20 \mathrm{~mm} * 20 \mathrm{~mm} * 0.5 \mathrm{~mm}$ represents a noise source such as the output (switching node) trace inside the switching cell, constantly generating impulse voltage waves. A larger copper sheet of size $50 \mathrm{~mm} * 50 \mathrm{~mm} * 0.5 \mathrm{~mm}$ represents the shield layer between circuitry and ground and a copper sheet of size $70 \mathrm{~mm} * 70 \mathrm{~mm} * 0.5 \mathrm{~mm}$ represents the grounded baseplate/heatsink. A return point of the same size as the noise source which represents the position where coupled current is returned, for example either potential of the DC-link. In this model the return point is electrically connected to the screen layer by a set of bus bars whose properties are chosen to represent interconnections such as a wire.

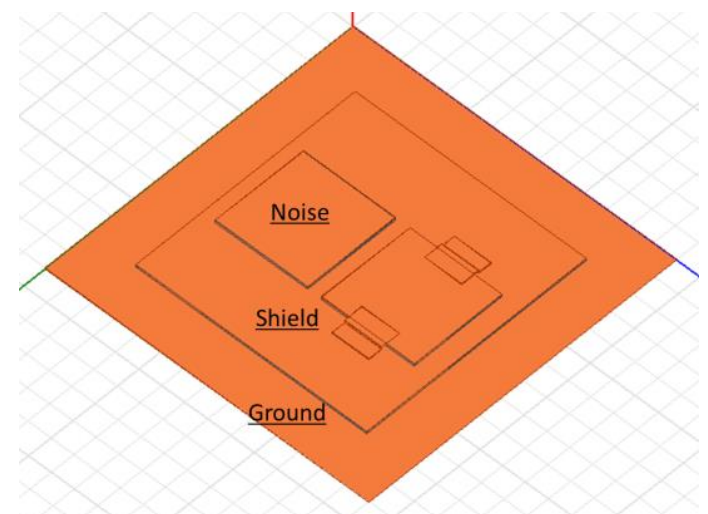

Figure.5 model of CM returning system within ANSYS MAXWELL simulation.

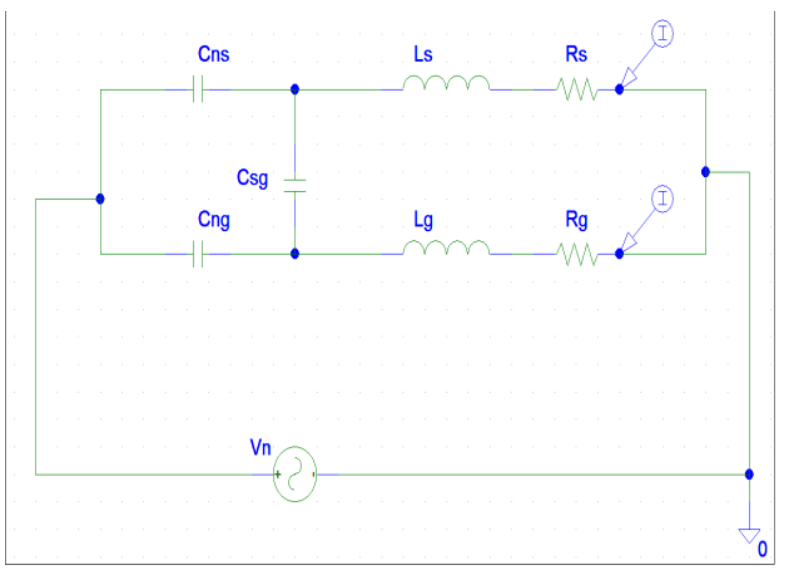

Figure.6 equivalent circuit of screen and return paths within Pspice simulation.

Polyimide layers of thickness $0.05 \mathrm{~mm}$ are inserted between each of the copper layers to represent the required electrical isolation. By creating an excitation point on the noise source and two sink points on the return pat and on the ground layer respectively, a complete screening system can be realized by this model and $\mathrm{CM}$ current returning function of screen layer can be investigated through the following simulations and measurements.

All parasitic capacitance, inductance and resistance of this model are extracted by ANSYS MAXWELL, and an equivalent circuit built within PSPICE simulation software, as shown in Fig.6. $V n$ is noise source; $C n s$ is capacitance between noise source and ground; $C n s$ is capacitance between noise source and screen layer; Csg is capacitance between screen and ground; $L s$ and $L g$ are inductance of the current path on screen layer and ground layer. $R s$ and $R g$ are resistance of current path on screen layer and ground layer respectively. By measuring the currents flowing on the screen and ground layer, the impedance of both current paths can be obtained. Impedance comparison between current paths on both screen layer and ground shown in Fig.7, 
with simulation frequency from $10 \mathrm{kHz}$ to $100 \mathrm{MHz}$. From the initial frequency to $10 \mathrm{MHz}$, the impedance on screen layer is 100 times less $(-40 \mathrm{~dB})$ than that on ground, and after then two traces tend to converge together. From this result we can understand, capacitance Cng and Cns mainly affect the impedance and dominate the impedance ratio at low frequency. As the inductances $L s$ and $L g$ begin to dominate at higher frequencies crosstalk $(C s g)$ between the two current path starts to affect the current ratio. As shown theoretically, $Y s$ and the ratio of coupled capacitance $\mathrm{Cng}$ and $\mathrm{Cns}$ dominate the current returning effect and $\mathrm{CM}$ screening effectiveness. Fig. 8 show that a reduced value of $L s$.(increased $Y s$ ) would improve CM screening results by pushing the resonant points to higher frequency and maintain difference of the impedance between $Z$ screen and $Z$ ground, which means the impedance of internal current returning loop to DC-link is small compared with that of the external ground path.

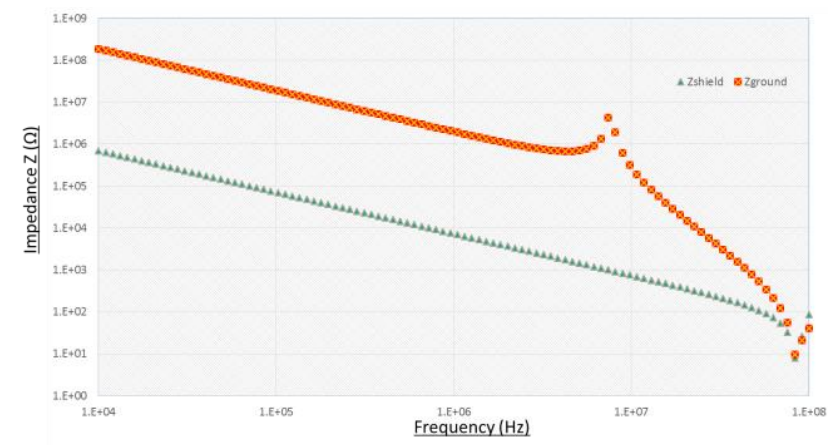

Figure.7 Impedance comparison between current paths of screen and ground.

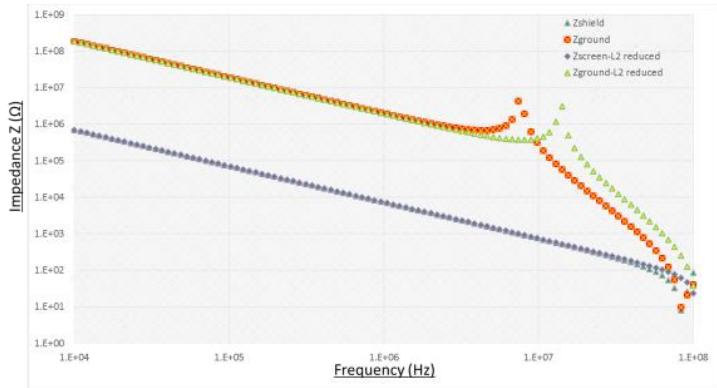

Figure. 8 Impedance comparison between current paths of screen and ground when Ls is reduced

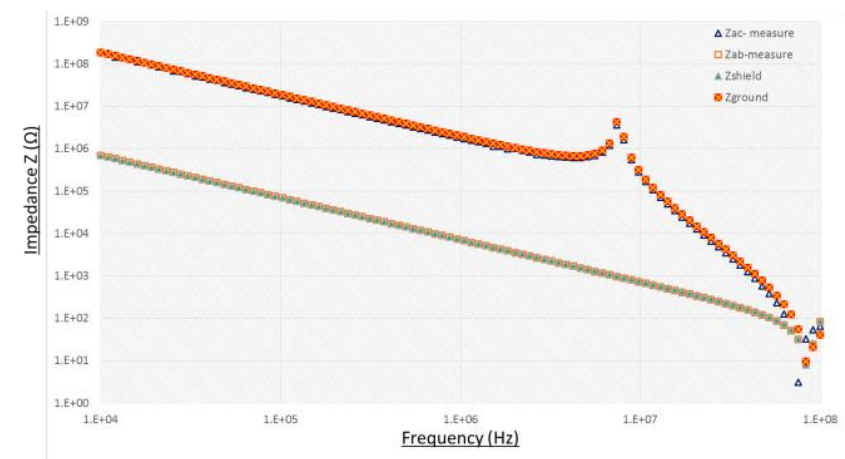

Figure.9 impedance comparison between simulation and impedance analyzer measurement.
We used impedance analyzer KEYSIGHT E4990A to measure impedance of internal and external current path $Z s$ and $Z g$, shown in Fig.9. The results from simulation and measurement aligns with each other very well, demonstrating that the equivalent circuit can well represent CM screen layer and can be used to investigate impact on current returning path with different screen arrangements. However at high frequency (above $50 \mathrm{MHz}$ ) a small deviation is visible. That is because at high frequency, lumped equivalent circuit might lose accuracy. Instead, a distributed circuit with multiple elements might be desirable to obtain better accuracy on characterizing screen layer at very high frequency.

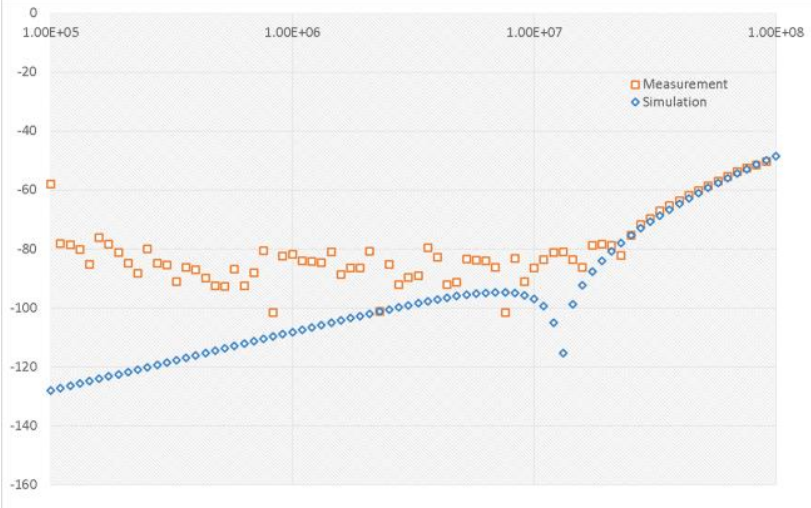

Figure.10 Noise attenuation between measurement and simulation results.

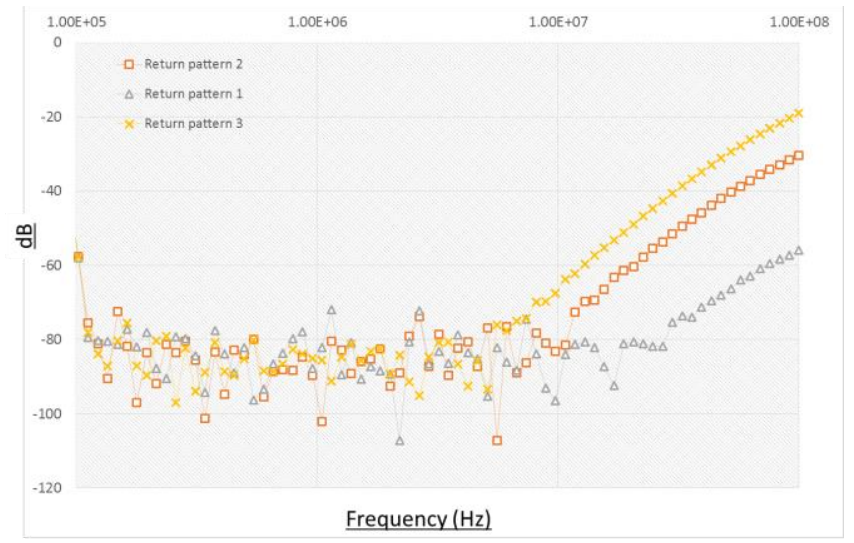

Figure.11 Noise attenuation through screen layer with different return patterns

We also use KEYSIGHT ENA Network Analyzer E5080A to measure signal losses between noise source (port 1) and ground (port 2) to investigate how much excited noise can spread to ground side. For CM screen layer, insertion voltage gain is the ratio of the port voltage at load side (port 2) without the screen layer to that with screen layer. Compared with the simulation results from PSPICE model, we can find that two result agree with each other very well, as shown in Fig.10. Xaxis is frequency from $100 \mathrm{kHz}$ to $100 \mathrm{MHz}$, and Y-axis is signal attenuation (Insertion Voltage Gain $\left[=-20 \log \left(\frac{V_{\text {with screen }}}{V_{\text {without screen }}}\right)\right]$ ). Noise floor from measurement background (about $-90 \mathrm{~dB}$ ) spreads from $100 \mathrm{kHz}$ to $10 \mathrm{MHz}$ and then insertion voltage gain goes up to $-20 \mathrm{~dB}$ at $100 \mathrm{MHz}$. On the other hand, measurement 
of noise attenuation with different return pattern were also conducted, and the results are shown in Fig. 11. Different return patterns produce different $Y S$ and result in different signal attenuation from noise source to ground. The trends in insertion voltage gain correspond to previous results measured by impedance analyzer. Lower impedance ratio of internal current path compared with external path always results in better screening effectiveness.

\section{B. Elctromagnetic shielding}

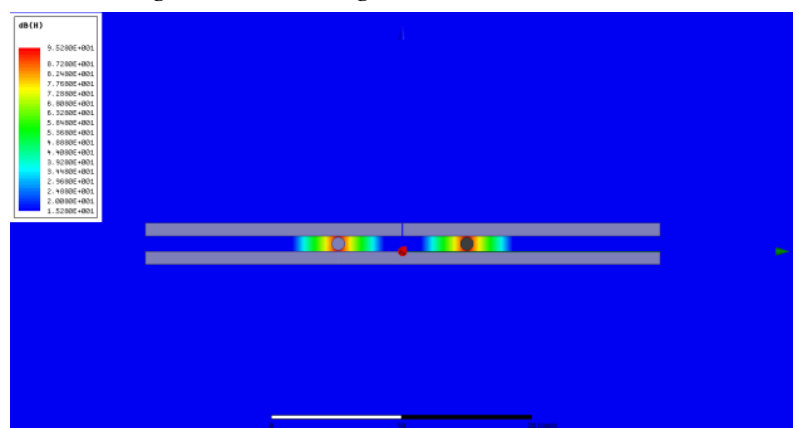

Figure.12 Magnetic field plot around shield layers for loop current source.

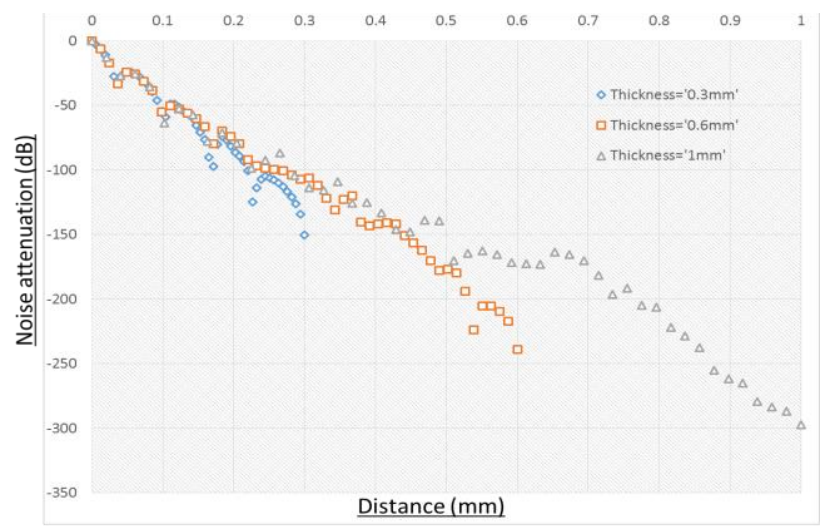

Figure.13 Magnetic field attenuation along cross-section with various thickness

From the theory mentioned above, generated varying magnetic field can be cancelled, diverted and contained within a good conductive shield layer. The shielding effectiveness is decided by the skin depth, distance from noise to shield layer, size and thickness of shield layer. A thicker shield layer with continuous design can provide massive magnetic field attenuation. We used software ANSYS MAXWELL to study magnetic field shieling effectiveness. The Maxwell 3D package, using eddy current solver method takes into account the eddy current effects, skin depth effect, and proximity effect encountered at high frequency. By considering current commutation within switching cell as a noise source, constantly generating radiative EMI noise, a simple model can be built within Maxwell 3D, and its magnetic field distribution is shown in Fig. 12. Noise source is inserted between two copper shield layers and most magnetic field is attenuated and contained between the shields. Fig.13 shows the noise attenuation through shield layers of different thickness $(0.3 \mathrm{~mm}$, $0.5 \mathrm{~mm}$ and $1 \mathrm{~mm})$. We can clearly see that the thicker layer can provide more noise attenuation.

\section{CONCLUSION}

In this paper, we have proposed and analyzed a CM current and magnetic field attenuation technique employing screening and shielding layers within a power switching cell. We used the simulation tool ANSYS Maxwell and circuit simulator PSpice for prediction and an impedance analyzer to verify the high frequency performance of the screen layer. Good agreement between a simple equivalent circuit model and measurements were obtained. A Network Analyzer was used to investigate screening effectiveness for different screen layer arrangements. Our results show that screening effeteness is closely related to the screen geometry and associated electrical return path within the switching cell. A low-impedance current return path is critical to minimizing noise transmission to ground over a wide frequency range. In general, electromagnetic screening and shielding provide an attractive method to achieve low EM emission levels and may eliminate the need for a separate common mode filter for some configurations.

\section{REFERENCES}

[1] H. Bishnoi, P. Mattavelli, R. Burgos and D. Boroyevich, "EMI Behavioral Models of DC-Fed Three-Phase Motor Drive Systems," 03 October 2013.

[2] S. Wang, F.C.Lee and W.G.Odendaal, "Improving the Performance of Boost PFC EMI Filters," in Proc. IEEE Applied Power Electronics Conference and Exposition, 2003.

[3] S. Qu and D. Y. Chen, "Mixed-mode EMI Noise and Its Implications to Filter Design in Offline Switching," in IEEE Transactions on Power Electronics, July 2002.

[4] L. D. Stevanovic, R. A. Beaupre, E. C. Delgado and a. A. V. Gowda, "Low inductance power module with blade connector," in Applied Power Electronics Conference and Exposition (APEC), 2010.

[5] E. Vagnon, P.-O. Jeannin, J.-C. Crebier and Y. Avenas, "A Bus-Bar-Like Power Module Based on Three-

Dimensional Power-Chip-on-Chip Hybrid Integration," in IEEE Transactions on Industry Applications, 2010.

[6] A. Domurat-Linde and E. Hoene, "Analysis and Reduction of Radiated EMI of Power Modules," in Integrated Power Electronics Systems (CIPS), 2012. 